\title{
RELACIÓN DEL ÍNDICE DE MASA CORPORAL CON FELICIDAD, AUTOESTIMA Y DEPRESIÓN EN ADULTOS JÓVENES
}

The relationship between body mass index and happiness,
self-esteem, and depression in young adults ${ }^{1}$

Graciela Cristina Avitia², Verónica Portillo Reyes ${ }^{3}$, Gilberto Reyes Leal ${ }^{4}$, Yolanda Loya Méndez

Fecha de recepción: 23 de enero del 2018

Fecha de aceptación: 09 de marzo del 2018

1- Por única ocasión, se aceptó este artículo con cuatro autores, ya que dos de ellos provienen de un área en la que el trabajo con equipos grandes es común.

2- Nacionalidad: Mexicana. Grado: Doctor. Especialización: Psicología. Adscripción: Universidad Autónoma de Ciudad Juárez. Correo electrónico: graciela.avitia@uacj.mx. (D) ORCID: http://orcid.org/0000-0003-3270-972X

3- Nacionalidad:Mexicana. Grado: Doctorado. Especialización: Psicología. Adscripción: Universidad Autónoma de Ciudad Juárez. Correo electrónico: vportill@uacj.mx. (D) ORCID: http://orcid.org/0000-0003-2238-8053

4- Nacionalidad:Mexicana. Grado: Doctorado. Especialización: Medicina. Adscripción: Universidad Autónoma de Ciudad Juárez. Correo electrónico: greyes@uacj.mx. (D) ORCID: http://orcid.org/0000-0001-9416-6818

5- Nacionalidad: Mexicana. Grado: Doctorado. Especialización: Medicina. Adscripción: Universidad Autónoma de Ciudad Juárez. Correo electrónico: yloya@uacj.mx. (i) ORCID: http://orcid.org/0000-0002-1199-4149 


\section{Resumen}

La obesidad es una enfermedad con repercusión psicológica, por lo que es importante reconocer los rasgos psicológicos y de comportamiento de quienes la padecen. El objetivo de este trabajo es analizar los niveles de felicidad, autoestima y depresión en individuos con normopeso (NP) y con sobrepeso/obesidad (SO). La muestra estuvo constituida por 151 alumnos de la Universidad Autónoma de Ciudad Juárez (UACJ) con una media de edad de 21.44 años. Los resultados mostraron que el grupo de SO tuvo puntuaciones significativamente más elevadas en sentido positivo de la vida y realización personal, en comparación con el grupo de NP. Sin embargo, no se encontraron diferencias significativas en autoestima y depresión. Estos resultados no coinciden con los de otras investigaciones y podrían ser explicados por el grado de aceptación en el entorno social hacia las personas con obesidad, las estrategias de afrontamiento y la prevalencia de obesidad y sobrepeso entre otras variables con las que se ha asociado la relación obesidad/infelicidad.

Palabras clave: IMC, obesidad, felicidad.

\section{Abstract}

Obesity is a condition with psychological repercussions, hence the importance of identifying psychological and behavioral traits of those afflicted by it. The objective of this work is to analyze happiness, depression, and self-esteem levels in people with normal weight (NP) and overweight/obesity (SO). The sample was comprised of 151 students from the Universidad Autónoma de Ciudad Juárez (UACJ) with an average age of 21.44 years. Results show a significantly higher score in positive sense of life and personal realization in the SO group in comparison with the NP group. Nonetheless, no significant difference was found in happiness and depression levels. These results part away from those of other existing research which might suggest that people with SO do not experience lack of acceptance from their social environment, a factor normally associated with unhappiness in people with obesity.

Keywords: BMI, obesity, happiness. 


\section{Introducción}

$\mathrm{L}$

a obesidad es una enfermedad (Calañas-Continente, Arrizabalaga, Caixàs et al., 2010) definida como la acumulación excesiva de grasa corporal, que puede perjudicar la salud (Organización Mundial de la Salud [OMS], 2016). Actualmente México ocupa el primer lugar en sobrepeso y el segundo en obesidad a nivel mundial (Organization for Economic Cooperation and Development [OECD], 2017). Esto es alarmante puesto que las consecuencias de la obesidad son numerosas, no solo en el ámbito económico, sino en los aspectos físico y psicológico (Yumuk, Frühbeck, Oppert et al., 2014).

Entre los rasgos psicológicos que se han asociado a la obesidad se encuentran inadecuada calidad de vida y depresión (Ciangura, Carette, Faucher et al., 2017), sentimientos de temor, percepción negativa de la imagen corporal, inseguridad, tristeza, infelicidad, (Luppino, de Wit, Bouvy et al., 2010), ansiedad, alteraciones emocionales y baja autoestima (Degirmenci, Kalkan-Oguzhanoglu, Sozeri-Varma et al., 2015).

La felicidad se considera un estado afectivo de satisfacción plena que experimenta subjetivamente el individuo en posesión de un bien anhelado (Alarcón, 2006), también descrita como la percepción intrínseca de bienestar (Seguí, 2017). Para Diener (2013), el bienestar subjetivo o bienestar psicológico equivale a ser feliz y se refiere a la evaluación afectiva y cognitiva que hacen las personas de su propia vida.

La felicidad es consecuencia de la buena salud, amor, comodidad y libertad, sin embargo, estas condiciones no son determinantes definitivas ya que la felicidad es subjetiva, depende de la condición social, cultura, religión y va vinculada a una apreciación personal (Seguí, 2017). Además, ésta es influenciada por el ambiente (Warr, 2013), las relaciones familiares y la personalidad (Morán, Fínez y Fernández-Abascal, 2017). Por otra parte, se sabe que la infelicidad puede repercutir negativamente en la calidad de vida. En un estudio realizado por Vera, Velasco, Grubits et al. (2016) se encontró que las personas infelices en comparación con las felices sienten que tienen menos logros, menor reconocimiento y menos redes de apoyo.

Estudios que analizaron la asociación entre el índice de masa corporal (IMC) y la felicidad han mostrado que a mayor IMC es menor el nivel de felicidad, pero solamente cuando en el entorno social el sobrepeso y la obesidad se perciben negativamente (Pinhey et al., como se citó en Cornelisse-Vermaat, Antonides, Van Ophem et al., 2006). En otro estudio se evidenció que las personas con obesidad eran menos felices que las personas con normopeso, encontrando como indicador que la infelicidad asociada a la obesidad es más común en entornos donde la obesidad es menos prevalente (Graham, 2008). Coincidiendo con este, estudios más recientes como el de Caixàs y Lecube (2014), demuestran que las personas que padecen obesidad presentan una menor calidad de vida, pero sólo en países en donde ésta condición no es tan común.

En estudios recientes se reporta que los individuos con obesidad muestran menores niveles de bienestar psicológico en aspectos como propósito en la vida y crecimiento personal (Magallares, Benito, Irles et al., 2014). Se ha encontrado también que la obesidad guarda una relación inversamente proporcional con la satisfacción personal, es decir, a mayor obesidad menor satisfacción personal (Wadsworth y Pendergast, 2014). Existe además evidencia que muestra un menor crecimiento personal en quienes presentan obesidad tipo dos (individuos con un IMC entre 30-34.9) y tres (IMC entre 35-40.9) comparado con las personas con sobrepeso y normopeso (Robertson, Davies y Winefield, 2015). 
El impacto de la obesidad en el bienestar subjetivo o felicidad se debe a las consecuencias adversas que ésta ejerce sobre la salud y al desempeño general de las personas con obesidad en su entorno social (Böckerman, Johansson, Saarni et al., 2014). La asociación entre la infelicidad y la obesidad también se ha atribuído a la percepción de un ideal inalcanzable de la imagen corporal. Se puede concluir que la insatisfacción con la propia imagen corporal y ser sujeto de burlas es lo que determina la felicidad en las personas con obesidad (Guzmán y Lugli, 2009). En este mismo contexto se ha observado que la discriminación hacia las personas con obesidad determina en ellas una menor satisfacción con la vida (Magallares et al., 2014). En adolescentes se observan resultados similares en la relación obesidad/satisfacción y se atribuyen a factores como la percepción de sí mismos, de sus compañeros y de sus padres.

La obesidad se relaciona también con la autoestima, que se define como la autoevaluación que tiene de sí mismo un individuo (Sánchez y Ruiz, 2015). En este sentido, resultados recientes indican que no sólo la autoestima, sino el estado físico y la vida social mejoraron en sujetos después de bajar de peso, mediante cirugía bariátrica (Bertheuil, Thienot, Chaput et al., 2015). Estudios en niños (Ortega, Joaquin, Palomares et al., 2015) y en universitarios (Aguilar-Ye, Puig-Sosa, Luna et al., 2002) también muestran evidencia de la relación que tiene la obesidad con problemas de autoestima. Otros estudios no han confirmado esta relación (Mäkinen, Lindberg, Komulainen et al., 2015).

La obesidad y la depresión muestran una estrecha relación causa-consecuencia, pues la depresión puede ser un factor importante para la obesidad, y la obesidad para la depresión (Vogelzangs, Kritchevsky, Beekman et al., 2010). Se ha encontrado que los sujetos con obesidad tienen mayores posibilidades (55\%) de presentar depresión en comparación con los sujetos de peso normal. Mientras que las personas depresivas tienen mayor riesgo (58\%) de padecer sobrepeso (Luppino et al., 2010). Otro estudio muestra que en adolescentes, la baja autoestima y depresión están relacionados con la insatisfacción corporal, además encontraron una relación entre sobrepeso percibido y baja autoestima, sin embargo, el sobrepeso no se relaciona con baja autoestima ni con depresión (Ozmen, Ozmen, Ergin et al., 2007). En su investigación, Robertson et al. (2015) encontraron que personas con obesidad tipo tres mostraron mayores niveles de depresión que los tipo dos, los de sobrepeso y normopeso.

En resumen, existe una amplia variedad de estudios sobre el bienestar psicológico en general, sin embargo, son pocos los que se centran específicamente en la relación de felicidad, autoestima y depresión con la obesidad. Dirigir la atención al estudio de estas variables para crear estrategias psicológicas de intervención, puede ser de utilidad en el enfoque integral de la obesidad. El objetivo de este estudio fue medir los niveles de felicidad, autoestima y depresión en individuos con normopeso y sobrepeso/obesidad.

\subsection{Método 1.2. Diseño}

Se diseñaron dos cuasiexperimentos de tipo no experimental descriptivos para comparar grupos independientes. Para el primer diseño se dividió a la muestra por normopeso y sobrepeso/obesidad de acuerdo con el IMC. En el segundo, tomando en cuenta la circunferencia de cintura (CC) se comparó a los participantes que presentaron riesgo cardiovascular con los que no presentaron. 


\subsection{Muestra}

La muestra inicial de este proyecto fue de 151 alumnos del Instituto de Ciencias Biomédicas (ICB) de la UACJ de las carreras de veterinaria, nutrición y entrenamiento deportivo. La muestra final fue de 145 alumnos debido a que tres sujetos no completaron los procedimientos del proyecto y otros tres fueron excluidos por presentar bajo peso. Los participantes tuvieron una media de edad de 21.44 (DE \pm 3.15 ), El 41.4\% fueron hombres y el $58.6 \%$ mujeres; el $52.2 \%$ presentó peso normal, el $26.9 \%$ sobrepeso y el $17.9 \%$ obesidad.

\subsection{Instrumentos}

Escala de felicidad de Alarcón (2006), constituida por 27 reactivos tipo Likert con cinco alternativas de respuesta (desde totalmente de acuerdo hasta totalmente en desacuerdo). Esta escala se compone por 4 factores: sentido positivo de la vida, satisfacción con la vida, realización personal y alegría de vivir. La escala tiene un Alfa de Cronbach de 916 lo que indica una buena consistencia interna.

Escala de Autoestima de Rosenberg (1965) cuenta con 10 reactivos formato Likert con 4 opciones de respuesta (desde muy de acuerdo hasta muy en desacuerdo). Cinco de los reactivos están redactados positivamente y los otros cinco negativamente. La calificación de la escala es que a mayor puntuación mejor autoestima. Esta escala presenta una fiabilidad de .80 .

Escala de Depresión de Zung (1965) tiene 20 reactivos tipo Likert con 4 opciones de respuesta (desde poco tiempo hasta la mayor parte del tiempo). Diez de los cuales están redactados positivamente y otros diez negativamente. La escala se caracteriza por incluir los 4 factores más comunes en la depresión: el efecto dominante, los equivalentes fisiológicos, otras perturbaciones y actividades psicomotoras. El Alfa de Cronbach de la escala se encuentra entre .79 y .92.

\subsubsection{Datos demográficos}

Se registró la edad, género, estado civil, carrera, si trabaja o no, nivel socioeconómico, actividad física realizada semanalmente y el número de amigos cercanos. El nivel socioeconómico (NSE, ver cuadro 1) se midió en base a la Asociación Mexicana de Agencias de Investigación de Mercado (AMAI, 2005).

\section{Cuadro 1.Nivel socioeconómico en México}

\begin{tabular}{|c|c|c|}
\hline NSE & Ingreso mínimo mensual & Ingreso máximo mensual \\
\hline A $/ B$ & $\$ 85,000.00+$ & \\
\hline C+ & $\$ 35,000.00$ & $\$ 84,999.00$ \\
\hline C & $\$ 11,600.00$ & $\$ 34,999.00$ \\
\hline D+ & $\$ 6,800.00$ & $\$ 11,599.00$ \\
\hline D & $\$ 2,700.00$ & $\$ 6,799.00$ \\
\hline E & \$ 0.00 & $\$ 2,699.00$ \\
\hline & Nota: NSE $=$ Nivel Socioeconómico. \\
& Fuente: AMAI (2005)
\end{tabular}




\subsection{Procedimiento}

La recolección de la muestra se realizó en el ICB, mediante invitación difundida en las aulas. Los criterios de inclusión incluyeron la firma de consentimiento informado que detallaba la descripción y características, procedimientos y riesgos de la investigación. Una vez aceptada su participación se les citó en la clínica de nutrición para realizar las diferentes etapas del proyecto (datos demográficos, escalas de felicidad, autoestima y depresión y el estudio antropométrico para obtener el IMC y la medida de la CC). Se mantuvo la confidencialidad de los datos, mediante el uso de folios. Se contó con la aprobación del comité de bioética de la Universidad Autónoma de Ciudad Juárez.

\subsubsection{Recolección de datos}

\subsubsection{Estudio antropométrico}

Se realizaron mediciones antropométricas como peso, talla y circunferencia de cintura según las especificaciones establecidas en el manual del Instituto Nacional de Salud Pública (Olaiz-Fernández, RiveraDommarco, Shamah-Levy et al., 2006). Para ello se utilizó una báscula digital y un estadímetro de pared, ambos marca Seca. Las Mediciones se realizaron por personal capacitado de la clínica de nutrición del ICB.

Además, se calculó el índice de masa corporal: $\left[\mathrm{IMC}=\operatorname{Peso}(\mathrm{k}) /\left(\right.\right.$ Talla $\left.(\mathrm{m})^{2}\right]$ y se clasificó a la población de estudio según este indicador en bajo peso, peso normal, sobrepeso y obesidad. Utilizando para ello los puntos de corte de la norma oficial mexicana (NOM) del 2010 para sobrepeso y obesidad. Se ubicó en bajo peso a los participantes cuyo IMC se encontraba por debajo de 18.5; normal dentro del rango de 18.5 a 25; sobrepeso entre 25 y 30; y obesidad por encima de 30.

\subsubsection{Circunferencia de cintura}

Se clasificó a los participante en dos grupos: sin riesgo y con riesgo cardiovascular según la medida de la circunferencia de la cintura, utilizando como puntos de corte los establecidos por la Asociación Latinoamericana de Diabetes (Rosas, Calles, Friege et al., 2009). Considerando de bajo riesgo cardiovascular valores por debajo de $88 \mathrm{~cm}$ en las mujeres y de 94 en los varones.

\subsection{Análisis de datos}

Para describir a la muestra se realizaron análisis de frecuencias y porcentajes de las variables. Mediante la prueba t de Student para grupos independientes, se compararon las medias de felicidad, autoestima y depresión de los grupos divididos por el IMC y por la CC. Para corroborar que las variables demográficas tomadas en cuenta en este estudio no afectan a los resultados se llevaron a cabo análisis de covarianza (ANCOVA). Se midió el tamaño de efecto de los análisis estadísticos mediante la $d$ de Cohen. 


\subsection{Resultados}

\subsection{Descriptivos}

De acuerdo con las variables demográficas, los análisis mostraron que hubo mayor frecuencia de mujeres, de solteros, de la carrera de veterinaria, de quienes no trabajan, de quienes tienen un nivel socioeconómico tipo $\mathrm{D}+\mathrm{y}$ de quienes realizaban menos de 150 minutos de actividad física a la semana. Las frecuencias y porcentajes de estas variables se muestran en el cuadro 2 . En cuanto al IMC y la CC, se encontró mayor frecuencia de las personas con normopeso y de quienes no presentaron riesgo cardiovascular respectivamente. Además, la media del IMC se ubicó dentro del rango de sobrepeso (cuadro 3).

Cuadro 2. Datos demográficos

\begin{tabular}{|c|c|c|c|c|c|}
\hline Datos demográficos & Frecuencia & Porcentaje & Datos demográficos & Frecuencia & Porcentaje \\
\hline $\begin{array}{l}\text { Sexo } \\
\text { Hombre } \\
\text { Mujer }\end{array}$ & $\begin{array}{l}60 \\
85\end{array}$ & $\begin{array}{l}41.40 \\
58.60\end{array}$ & $\begin{array}{c}\text { Trabaja } \\
\text { Sí } \\
\text { No }\end{array}$ & $\begin{array}{l}51 \\
92\end{array}$ & $\begin{array}{l}35.70 \\
64.30\end{array}$ \\
\hline $\begin{array}{c}\text { Estado Civil } \\
\text { Soltero } \\
\text { Casado } \\
\text { Unión libre }\end{array}$ & $\begin{array}{c}125 \\
12 \\
7\end{array}$ & $\begin{array}{c}86.88 \\
8.30 \\
4.90\end{array}$ & $\begin{array}{l}\text { Actividad física } \\
<150 \text { minutos } \\
150 \text { minutos } \\
>150 \text { minutos }\end{array}$ & $\begin{array}{l}78 \\
25 \\
41\end{array}$ & $\begin{array}{c}53.80 \\
17.20 \\
29.1\end{array}$ \\
\hline $\begin{array}{c}\text { Carrera } \\
\text { Médico veterinario } \\
\text { Nutrición } \\
\text { entrenamiento } \\
\text { deportivo }\end{array}$ & $\begin{array}{l}78 \\
41 \\
26\end{array}$ & $\begin{array}{l}53.80 \\
28.30 \\
17.90\end{array}$ & $\begin{array}{c}\text { NSE } \\
\text { A/B } \\
\text { C+ } \\
\text { C } \\
\text { D+ } \\
\text { D } \\
\text { E }\end{array}$ & $\begin{array}{c}3 \\
9 \\
35 \\
40 \\
4 \\
7\end{array}$ & $\begin{array}{c}2.20 \\
6.60 \\
25.70 \\
29.40 \\
30.90 \\
51.10\end{array}$ \\
\hline
\end{tabular}

Nota. NSE = Nivel socioeconómico. Los datos representados son con una muestra de 145 participantes. Se excluyeron a quienes que no completaron los procedimientos y a quienes presentaron bajo peso.

\section{Cuadro 3. Determinaciones antropométricas}

\begin{tabular}{|c|c|c|c|c|c|c|}
\hline & Frecuencia & Porcentaje & Mínimo & Máximo & Media & $\begin{array}{c}\text { Desviación } \\
\text { estándar }\end{array}$ \\
\hline $\begin{array}{c}\text { Índice de masa corporal } \\
\text { Bajo peso }\end{array}$ & 3 & 2.0 & & & \\
Normal & 80 & 53.0 & 17.19 & 40.39 & 25.16 & 4.85 \\
Sobrepeso & 39 & 25.8 & 17.2 & & & \\
Obesidad & 26 & 82.1 & 59.00 & 120.00 & 79.26 & 12.23 \\
\hline $\begin{array}{c}\text { Circunferencia de la cintura } \\
\text { (cm) }\end{array}$ & 124 & 15.9 & & & \\
\hline Sin riesgo & 24 & & 42.00 & 120.40 & 70.70 & 16.26 \\
\hline
\end{tabular}

Nota. Los datos representados son con una muestra de 148 participantes. Se excluyeron a los que no terminaron el proyecto y están incluidas aquellas con bajo peso. 


\subsection{Prueba t de student}

En los grupos divididos por el IMC los resultados mostraron únicamente diferencias estadísticamente significativas en el factor de sentido positivo de la vida $[\mathrm{t}(141)=-2.07, \mathrm{p}<.05, \mathrm{~d}=.35$ ] y en el factor de realización personal $[\mathrm{t}(142)=-2.48, \mathrm{p}<.05, \mathrm{~d}=.42$ ] de la escala de felicidad. En ambos casos el grupo de $\mathrm{S} / \mathrm{O}$ tuvo una media mayor que el grupo de NP, sugiriendo que los universitarios con sobrepeso y obesidad tienen un mejor sentido positivo de la vida y una mejor realización personal que los universitarios con normopeso. De acuerdo con la d de Cohen, estas diferencias fueron moderadas (ver cuadro 4).

En los grupos divididos por la CC no se encontraron diferencias estadísticamente significativas en las variables estudiadas.

\section{Cuadro 4. Pruebas t de Student entre los grupos según el IMC}

\begin{tabular}{|c|c|c|c|c|c|c|c|}
\hline \multirow{2}{*}{ Variables } & \multicolumn{2}{|c|}{ Normopeso } & \multicolumn{2}{|c|}{ Sobrepeso/Obesidad } & \multirow{2}{*}{$\mathbf{t}$} & \multirow{2}{*}{$\mathbf{p}$} & \multirow{2}{*}{$\begin{array}{l}\text { d de } \\
\text { Cohen }\end{array}$} \\
\hline & Media & $\begin{array}{l}\text { Desviación } \\
\text { estándar }\end{array}$ & Media & $\begin{array}{l}\text { Desviación } \\
\text { estándar }\end{array}$ & & & \\
\hline $\begin{array}{l}\text { Sentido positivo de } \\
\text { la vida }\end{array}$ & 18.00 & 6.57 & 20.80 & 9.53 & -2.07 & .04 & .35 \\
\hline $\begin{array}{l}\text { Satisfacción con la } \\
\text { vida }\end{array}$ & 11.62 & 3.88 & 12.27 & 4.11 & -.96 & .34 & - \\
\hline Realización personal & 13.73 & 2.89 & 14.98 & 3.15 & -2.48 & .01 & .42 \\
\hline Alegría de vivir & 6.53 & 2.27 & 7.06 & 2.89 & -1.23 & .22 & - \\
\hline Felicidad total & 48.47 & 14.60 & 53.87 & 19.11 & -1.91 & .06 & - \\
\hline Depresión & 35.40 & 7.81 & 36.17 & 7.57 & -.60 & .55 & - \\
\hline Autoestima & 16.30 & 4.11 & 17.38 & 6.05 & -1.28 & .20 & - \\
\hline
\end{tabular}

Nota: Según el test de Levene, se asumen varianzas iguales en los factores significativos

\subsection{Análisis de Covarianza}

Las covariables tomadas en cuenta para los ANCOVAS fueron el sexo, edad, estado civil, si trabaja o no, nivel socioeconómico y actividad física. Los resultados fueron, en sentido positivo de la vida [F (1, $126)=3.94, \mathrm{p}<.05, \mathrm{~d}=.29]$ y en realización personal $[\mathrm{F}(1,179)=5.66, \mathrm{p}<.05, \mathrm{~d}=.39]$ (cuadro 5). Estos resultados son consistentes con los de las pruebas $t$, lo que sugiere que las covariables no tienen un efecto sobre el IMC, es decir, las diferencias encontradas en sentido positivo de la vida y en realización personal siguen siendo estadísticamente significativos independientemente de dichas covariables (si éstas se mantienen constantes). 


\section{Cuadro 5. ANCOVAS entre los grupos según el IMC}

\begin{tabular}{|c|c|c|c|c|c|c|c|}
\hline & \multicolumn{2}{|c|}{ Normopeso } & \multicolumn{2}{|c|}{ Sobrepeso/Obesidad } & \multirow[b]{2}{*}{$\mathbf{F}$} & \multirow[b]{2}{*}{$\mathbf{p}$} & \multirow{2}{*}{$\begin{array}{l}\text { d de } \\
\text { Cohen }\end{array}$} \\
\hline & Media & $\begin{array}{l}\text { Desviación } \\
\text { estándar }\end{array}$ & Media & $\begin{array}{l}\text { Desviación } \\
\text { estándar }\end{array}$ & & & \\
\hline $\begin{array}{l}\text { Sentido positivo de la } \\
\text { vida }\end{array}$ & 18.11 & 6.57 & 20.47 & 9.84 & 3.94 & .049 & .29 \\
\hline Realización personal & 13.75 & 2.92 & 14.95 & 3.19 & 5.66 & .019 & .39 \\
\hline
\end{tabular}

\section{Conclusión}

Este trabajo tuvo como objetivo medir y comparar los niveles de felicidad, autoestima y depresión entre los individuos con peso normal y con sobrepeso y obesidad. Estudios anteriores indican que las personas con obesidad tienden a presentar mayores niveles de depresión y menores niveles de felicidad y autoestima. Sin embargo, no fue así en los resultados obtenidos en este estudio, donde se encontró que las personas con sobrepeso/obesidad sienten mayor realización personal y tienen un mejor sentido positivo de la vida (factores que describen la felicidad) que las personas con normopeso. No se encontraron diferencias estadísticamente significativas entre los grupos según el IMC y la CC con la depresión y autoestima.

Trabajos anteriores sustentan que las personas con obesidad y sobrepeso tienen niveles menores de felicidad, autoestima y bienestar psicológico. Esto se atribuye principalmente a las burlas de las que son objeto estas personas (Ciangura et al., 2016) a la autopercepción de la imagen corporal, a la percepción que de ellos tienen las demás personas (Almenara, Aimé, Maïano et al., 2017) y a la posible discriminación por su peso (Magallares et al., 2014). En este estudio se encontró lo contrario, el grupo de SO mostró tener un mejor sentido positivo de la vida y una mayor realización personal que las personas con NP.

Estos resultados pueden indicar que no en todos los contextos sociales o culturales las personas con obesidad son menos felices y que esto más bien es el resultado de un entorno en el que la obesidad es poco prevalente o considerada un aspecto negativo. Lo que se refleja en conductas de discriminación o burlas dirigidas hacia quienes la presentan, alterando el estado de bienestar psicológico (Bassuk y Manson, 2017). Dado que México ocupa el segundo lugar a nivel mundial en obesidad y el primero en sobrepeso (OECD, 2017), se consideran el sobrepeso y la obesidad como estados comunes. De tal manera que en un entorno donde éstos son habituales es posible que las personas con obesidad no representen un grupo señalado, lo que podría explicar los resultados del estudio.

Un hallazgo interesante de este estudio es que el grupo de NP muestra niveles inferiores de felicidad. Una probable explicación se encuentra en la preocupación por su imagen corporal, el consumo de alimentos, la talla, los pensamientos de las demás personas si suben de peso, etc. Esta hipótesis se apoya en algunos estudios donde han encontrado que muchas jóvenes con peso normal tienen un fuerte deseo de estar más delgadas y tener menos grasa corporal, lo que puede indicar una constante preocupación por bajar de peso y como consecuencia menor bienestar psicológico (Míguez, de la Montaña, Fernández et al., 2009). Datos similares se mostraron en otro estudio que se llevó a cabo en 
una muestra de universitarios, donde las mujeres más obsesionadas por adelgazar son aquellas que se encuentran en el límite superior del normopeso, (Míguez, de la Montaña, González et al., 2011).

Otro estudio en adolescentes, donde las mujeres presentaron menor sobrepeso y obesidad, a la vez que mayor insatisfacción corporal y sensación de obesidad, apoya de igual manera la hipótesis de que la preocupación por la imagen corporal puede explicar los resultados obtenidos (Ramos, Rivera y Moreno, 2010). La constante preocupación de las personas con normopeso por estar más delgadas y la falta de satisfacción por su imagen corporal explicarían la menor puntuación en la escala de felicidad. En este contexto resultaría de interés ampliar este tipo de estudios incluyendo como variables de estudio aspectos como la imagen corporal, aceptación social y bullying. En relación con esto, un estudio reciente reporta que la satisfacción con la vida tiene que ver con la discrepancia del yo real y el yo ideal, a menor discrepancia mayor satisfacción con la vida (Schnettler, Miranda, Sepúlveda et al., 2014), Los estudios citados han encontrado inconformidad con la imagen corporal real, en relación con la ideal, en personas con peso normal. Lo que podría ser una línea de interés para identificar la razón de los resultados obtenidos en este estudio.

En contraste a los hallazgos descritos anteriormente, estudios realizados en niños (Warschburger, 2005) y adolescentes (Fararouei, Brown, Akbartabar et al., 2013), han encontrado que quienes presentan obesidad tienen menores niveles de felicidad. Estos estudios indicarían que los niños y adolescentes con obesidad son más susceptibles a las burlas y a la autopercepción negativa debido a la discriminación en su contexto social. Podría pensarse que los universitarios tienen mejores estrategias de afrontamiento ante estas situaciones, lo cual explicaría las mejores puntuaciones en la escala de felicidad que presenta el grupo de sobrepeso/obesidad, sin embargo, no explica los bajos niveles del grupo de normopeso.

Estos resultados no se pueden extrapolar al resto de la población, ya que tenemos como limitación que la muestra fue exclusivamente de estudiantes universitarios, por lo que es recomendable ampliar la investigación en población abierta para aumentar la consistencia de los resultados.

Para finalizar, los resultados de este estudio muestran que la población universitaria con normopeso presenta niveles más bajos de felicidad, por lo que es necesario explorar los factores que contribuyen a estos resultados. Sería interesante explorar la insatisfacción corporal, la autopercepción corporal y el deseo por estar más delgado para corroborar en esta sociedad los resultados obtenidos en estudios previos. También sería interesante comparar niños, adolescentes y jóvenes adultos y adultos, para conocer los grupos más susceptibles. Se podría considerar incluir otras variables que permitan explicar estos resultados.

A manera de conclusión, los resultados de este estudio dejan ver que, en sociedades como esta, en donde el sobrepeso y la obesidad son comunes, las personas con SO pueden no sentirse discriminadas y sí estar satisfechas consigo mismas, y por tanto más felices que en sociedades en donde estas condiciones no son la norma.

Por último, existe un dilema más que sería interesante abordar, ${ }_{¿}$ Es la falta de bienestar psicológico o infelicidad la causa o la consecuencia de la obesidad? Tal vez ésta sea una variable que influye determinantemente en los resultados de este tipo de estudios.

\section{Referencias}

Aguilar-Ye, Arturo, Pedro Puig-Sosa, Luis Luna, Pedro Sánchez, Roberto Rodríguez y Leoncio Rodríguez. 2002. La autoestima y su relación con el índice de masa corporal al culminar la adolescencia. Revista Mexicana de Pediátrica, 69(5): 190-193. 
Alarcón, Reynaldo. 2006. Desarrollo de una escala factorial para medir la felicidad. Revista Interamericana de Psicología, 40(1): 96-106.

Almenara, Carlos, Annie Aimé, Christopher Maïano, Anastasia Ejova, Guylaine Guèvremont, Chantal Bournival y Marie-Michèle Ricard. 2017. Weight stigmatization and disordered eating in obese women: The mediating effects of self-esteem and fear of negative appearance evaluation. Revue Européenne de Psychologie Appliquée/European Review of Applied Psychology, 67(3): 155-162. DOI: https://doi. org/10.1016/j.erap.2017.02.004

Asociación Mexicana de Agencias de Investigación de Mercado. 2005. Avances AMAI: Distribución de niveles socioeconómicos en el México urbano. https://jorgecardenas.files.wordpress.com/2007/11/art_ nse_amai.pdf (25 de enero, 2016)

Bassuk, Shari y JoAnn Manson. 2017. Obesity/overweight: Health consequences. International Encyclopedia of Public Health, 2da edición. 5: 277-294. DOI: http://dx.doi.org/10.1016/B978-0-12-8036785.00308-8

Bertheuil, Nicolas, Sophie Thienot, Benoit Chaput y Eric Watier. 2015. Quality-of-life assessment after medial thighplasty in patients following massive weight loss. Plastic and Reconstructive Surgery, 135(1): 67-73. DOI: https://doi.org/10.1097/PRS.0000000000000771 .

Böckerman, Petri, Edvard Johansson, Samuli Saarni y Suoma Saarni. 2014. The negative association of obesity with subjective well-being: is it all about health? Journal of Happiness Studies, 15(1): 857-867. DOI: https://doi.org/10.1007/s10902-013-9453-8

Caixàs, Assumpta y Albert Lecube. 2014. Where are obese people happier? Endocrinología y Nutrición, 61(1): 1-2. DOI: https://doi.org/10.1016/j.endonu.2013.10.003

Calañas-Continente, Alfonso, Juan Arrizabalaga, Assumpta Caixàs y Fernando Cordido. 2010. Recomendaciones diagnósticas y terapéuticas en el sobrepeso y la obesidad durante la adolescencia. Medicina Clínica, 135(6): 265-73. DOI: https://doi.org/10.1016/j.medcli.2009.02.041

Ciangura, Carlos, Claire Clarette, Pauline Faucher, Sebastien Czernichow y Jean Oppert. 2017. EMC Tratado de medicina, 21(2): 1-10. DOI: https://doi.org/10.1016/S1636-5410(17)84245-8

Cornelisse-Vermaat, Judith, Gerrit Antonides, Johan Van Ophem y Henriette Maassen. 2006. Body mass index, perceived health, and happiness: their determinants and structural relationships. Social Indicators Research, 79(1): 143-158. DOI: https://doi.org/10.1007/s11205-005-4097-7

Degirmenci, Taner, Nalan Kalkan-Oguzhanoglu, Gülfizar Sozeri-Varma, Osman Ozdel y Semin Fenkci. 2015. Psychological symptoms in obesity and related factors. Archives of Neuropsychiatry, 52(1): 42-46. DOI: https://doi.org/10.5152/npa.2015.6904

Diener, Ed. 2013. The remarkable changes in the science of subjective well-being. Perspectives on Psychological Science, 8(6): 663-666. DOI: https://doi.org/10.1146/annurev.psych.54.101601.145056

Fararouei, Mohammad, Ian Brown, Mehdi Akbartabar, Roksana Estakhrian y Jamileh Jafari. 2013. Happiness and health behaviour in Iranian adolescent girls. Journal of Adolescence, 36(6): 1187-1192. DOI: https://doi.org/10.1016/j.adolescence.2013.09.006

Graham, Carol. 2008. Happiness and health: lessons-and questions--for public policy. Health Affairs (Project Hope), 27(1): 72-87. DOI: https://doi.org/10.1377/hlthaff.27.1.72

Guzmán, Rosana y Zoraide Lugli. 2009. Obesidad y satisfacción con la vida: Un análisis de trayectoria. Suma Psicológica, 16(2): 37-50. 
Luppino, Floriana, Leonore de Wit, Paul Bouvy, Theo Stijnen, Pim Cuijpers, Brenda Penninx y Frans Zitman. 2010. Overweight, obesity, and depression: A systematic review and meta-analysis of longitudinal studies. Formerly Archives of General Psychiatry, 67(3): 220-229. DOI: https://doi.org/10.1001/archgenpsychiatry.2010.2.

Magallares, Alejandro, Pilar Benito, José Irles, Patricia Bolaños-Ríos e Ignacio Jauregui. 2014. Psychological well-being in a sample of obese patients compared with a control group. Nutrición Hospitalaria, 30(1): 32-6. DOI: https://doi.org/10.3305/nh.2014.30.1.7515

Mäkinen, Mauno, Nina Lindberg, Erkki Komulainen, Leena-Riitta Puukko-Viertomies, Veikko Aalberg y Mauri Marttunen. 2015. Psychological well-being in adolescents with excess weight. Nordic Journal of Psychiatry, 69(5): 354-363. DOI: https://doi.org/10.3109/08039488.2014.986194

Míguez, Monserrat, Julia de la Montaña, Josefina González y María González. 2011. Concordancia entre la autopercepción de la imagen corporal y el estado nutricional en universitarios de Orense. Nutrición Hospitalaria, 26(3): 472-479. DOI: https://doi.org/10.3305/nh.2011.26.3.4604

Míguez, Monserrat, Julia de la Montaña, Isasi Fernández, María González y Josefina González. 2009. Evaluación de la distorsión de la imagen corporal en universitarios en relación a sus conocimientos de salud. Nutrición clínica y dietética hospitalaria, 29(2): 15-23.

Morán, María, María, Fínez y Enrique Fernández-Abascal. 2017. Sobre la felicidad y su relación con tipos y rasgos de personalidad. Clínica y Salud, 28: 56-63. DOI: http://dx.doi.org/10.1016/j.clysa.2016.11.003 NORMA Oficial Mexicana NOM-008-SSA3-2010. 2010. Para el tratamiento integral del sobrepeso y la obesidad. http://dof.gob.mx/nota_detalle.php?codigo $=5154226 \& f e c h a=04 / 08 / 2010$ (10 de enero, 2016). Olaiz-Fernández, Gustavo, Juan Rivera-Dommarco, Teresa Shamah-Levy, Rosalba Rojas, Salvador Villalpando-Hernández, Mauricio Hernández y Jaime Sepúlveda. 2006. Encuesta Nacional de Salud y Nutrición. 2006. Cuernavaca, México: Instituto Nacional de Salud Pública.

Organization for Economic Cooperation and Development. 2017. Health at a glance 2017: OECD Indicators. https://www.oecd.org/mexico/Health-at-a-Glance-2017-Key-Findings-MEXICO-in-Spanish. pdf (20 de febrero, 2018).

Organización Mundial de la Salud. 2016. Obesidad y Sobrepeso. http://www.who.int/media centre/ factsheets/fs311/es/ (10 de febrero de 2016).

Ortega, María, José Muros, Juan Palomares, José Martín y María Cepero. 2015. Influence of BMI on selfesteem of children aged 12-14 years. Anales de Pediatría, 83(5): 311-317.

Ozmen, Dilek, Erol Ozmen, Dilek Ergin, Aynur Cakmakci, Nesrin Sen, Erbay Dundar y Oryal Taskin. 2007. The association of self-esteem, depression and body satisfaction with obesity among Turkish adolescents. BioMed Central Public Health, 7: 15-23. DOI: https://doi.org/10.1186/1471-2458-7-80

Ramos, Pilar, Francisco, Rivera y Carmen Moreno. 2010. Diferencias de sexo en imagen corporal, control de peso e índice de masa corporal de los adolescentes españoles. Psicothema, 22(1): 77-83.

Robertson, Sharib, Matthew Davies y Helen Winefield. 2015. Why weight for happiness? Correlates of BMI and SWB in Australia. Obesity Research \& Clinical Practice, 9(6): 609-612. DOI: https://doi. org/10.1016/j.orcp.2015.04.011

Rosas, Juan y Jorge Calles. 2009. Consenso de Prediabetes: Documento de posición de la Asociación Latinoamericana de Diabetes (ALAD). Revista de la Asociación Latinoamericana de Diabetes, 17(4): 146-158. Rosenberg, Morris. 1965. Society and the adolescent self-image. Princeton, NJ: Princeton University Press. 
Sánchez, Jaime y Ana Ruiz. 2015. Relación entre autoestima e imagen corporal en niños con obesidad. Revista Mexicana de Trastornos Alimentarios, 6(1): 38-44. DOI: https://doi.org/10.1016/j.rmta.2015.05.006 Schnettler, Berta, Horacio Miranda, José Sepúlveda, Ligia Orellana, Marianela Denegri, Marcos Mora y Germán Lobos. 2014. Variables que influyen en la satisfacción con la vida de personas de distinto nivel socioeconómico en el sur de Chile. Suma Psicológica, 21(1): 54-62. DOI: https://doi.org/10.1016/S01214381(14)70007-4

Seguí, Mateu. 2017. ¿Influye la felicidad en la mortalidad - Does happiness influence mortality? Medicina de Familia. SEMERGEN, 43(3): 232-233. DOI: http://dx.doi.org/10.1016/j.semerg.2016.04.007

Vera, José, Francisco Velasco, Heloisa Grubits e Iván Salazar. 2016. Parámetros de inclusión y exclusión en los extremos de la felicidad y satisfacción con la vida en México. Acta de Investigación Psicológica, 6: 2395-2403. DOI: http://dx.doi.org/10.1016/j.aipprr.2016.06.002

Vogelzangs, Nicole, Stephen Kritchevsky, Aartjan Beekman, Gretchen Brenes, Anne Newman, Suzanne Satterfield, Kristine Yaffe, Tamara Harris y Brenda Penninx. 2010. Obesity and onset of significant depressive symptoms: results from a community-based cohort of older men and women. The Journal of clinical psychiatry, 71(4): 391-399. DOI: https://doi.org/10.4088/JCP.08m04743blu

Wadsworth, Tim y Philip Pendergast. 2014. Obesity (sometimes) matters: The importance of context in the relationship between obesity and life satisfaction. Journal of Health and Social Behavior, 55(2): 196-214. DOI: https://doi.org/10.1177/0022146514533347

Warr, Peter. 2013. Fuentes de felicidad e infelicidad en el trabajo: una perspectiva combinada. Revista de Psicología del Trabajo y de las Organizaciones. 29(3). DOI: https://doi.org/10.5093/tr2013a15

Warschburger, Petra. 2005. The unhappy obese child. International Journal of Obesity, 29(2): S127-S129. DOI: https://doi.org/10.1038/sj.ijo.0803097

Yumuk, Volkan, Gema Frühbeck, Jean Oppert, Euan Woodward y Hermann Toplak. 2014. An EASO position statement on multidisciplinary obesity management in adults. Obesity Facts, 7(2): 96-101. DOI: https://doi.org/10.1159/000362191

Zung, William. 1965. A self-rating depression scale. Archives of General Psychiatry 12(1): 63-70. DOI: https://doi.org/10.1001/archpsyc.1965.01720310065008 